\title{
Renal Resistive Index as a Predictor of Acute Kidney Injury and Mortality in COVID-19 Critically III Patients
}

\author{
Edgar Garcia Cruz ${ }^{a} \quad$ Blanca Estela Broca Garcia ${ }^{c}$ Daniel Manzur Sandoval ${ }^{a}$ \\ Rodrigo Gopar-Nieto ${ }^{c}$ Francisco Javier Gonzalez Ruiz ${ }^{a}$ Linda Diaz Gallardo ${ }^{d}$ \\ Claudio Ronco ${ }^{\text {Magdalena Madero }}{ }^{b}$ Enzo Vasquez Jimenez ${ }^{b}$ \\ aDepartment of Cardiovascular Critical Care, Nacional Institute of Cardiology "Ignacio Chavez", Mexico City, Mexico; \\ bepartment of Nephrology, Nacional Institute of Cardiology "Ignacio Chavez", Mexico City, Mexico; 'Department \\ of Cardiology, Nacional Institute of Cardiology "Ignacio Chavez", Mexico City, Mexico; 'Department of Pediatrics, \\ Sanatorio Durango, Mexico City, Mexico; 'Department of Nephrology, San Bortolo Hospital and International Renal \\ Research Institute of Vicenza (IRRIV), Vicenza, Italy
}

\section{Keywords}

Renal resistive index · Acute kidney injury - Coronavirus disease $\cdot$ Renal replacement therapy

\begin{abstract}
Background: Acute kidney injury (AKI) in patients with COVID-19 can be caused by multiple mechanisms. Renal resistive index (RRI) is a noninvasive instrument to evaluate kidney hemodynamics, and it is obtained by analysis of intrarenal arterial waves using Doppler ultrasound. This study aimed to determine the role of RRI in predicting AKI and adverse outcomes in critically ill patients with COVID-19. Methods: This cross-sectional study included 65 patients with confirmed SARS-CoV-2 pneumonia admitted to the critical care unit from April 1, 2020, to June 20, 2020. Informed consent was obtained from all individual participants included in the study. Cardiac, pulmonary, and kidney ultrasonographic evaluations were performed in a protocolized way. Results: In this cohort, 65 patients were included, mean age
\end{abstract}

was 53.4 years, $79 \%$ were male, and $35 \%$ were diabetic. Thirty-four percent of patients developed $\mathrm{AKI}, 12 \%$ required RRT, and $35 \%$ died. Of the patients who developed AKI, $68 \%$ had $\mathrm{RRI} \geq 0.7$. Also, $75 \%$ of the patients who required RRT had RRI $\geq 0.7$. In the adjusted Cox model, the RRI $\geq 0.7$ was associated with higher mortality (HR 2.86, 95\% Cl: 1.19-6.82, $p=0.01$ ). Conclusions: Critical care ultrasonography is a noninvasive, reproducible, and accurate bedside method that has proven its usefulness. An elevated RRI may have a role in predicting AKI, RRT initiation, and mortality in patients with severe SARS-CoV-2 pneumonia.

(c) 2021 S. Karger AG, Basel

\section{Introduction}

In December 2019, a new respiratory disease called coronavirus disease-19 (COVID-19) caused by the SARSCoV-2 virus emerged in Wuhan City, Hubei Province, China, becoming a pandemic after spreading around the karger@karger.com

(c) 2021 S. Karger AG, Basel

www.karger.com/bpu

Karger $\stackrel{2}{=}$
Correspondence to:

Blanca Estela Broca Garcia, bebg888@gmail.com Enzo Vasquez Jimenez, enzo.vas.ji@gmail.com 
world. From the first descriptions, it was observed that the morbidity and mortality were not only due to respiratory complications [1], but also kidney involvement was frequent in COVID-19 in many manifestations [2]. Acute kidney injury (AKI) affects $20-40 \%$ of critically ill patients with COVID-19 [3].

AKI in patients with COVID-19 can be attributed to multiple mechanisms; the interrelation between the lungs and the kidneys is proposed; in addition, angiotensinconverting enzyme 2 serves SARS-CoV-2 as an input receptor for tubular epithelial cells in addition to pulmonary epithelial cells [4]. Furthermore, the cytokine release syndrome in COVID-19 has been associated with intrarenal inflammation, increased vascular permeability, volume depletion, and cardiomyopathy causing a cardiorenal syndrome type I [5]. Finally, there are nonspecific mechanisms such as hypovolemia, hypoxemia due to acute respiratory distress syndrome, and nephrotoxicity due to drugs and contrast media [6]. AKI can accelerate and perpetuate the inflammation that started in the lung, especially when there is an increase in central venous pressure, an increase in intrathoracic pressure, or water overload [7, 8].

Renal resistive index (RRI) is a noninvasive instrument to evaluate kidney hemodynamics, and it is obtained by analysis of intrarenal arterial waves using Doppler ultrasound [9]. In the Oracle study, it was shown that patients with right heart failure and elevated pulmonary artery systolic pressure had higher mortality. These patients have increased pressure in the right atrium, so it is expected that the RRI could be elevated; however, these have not been consistent [10]. This study aims to determine the relevance of RRI in predicting AKI and mortality in critically ill patients with COVID- 19 .

\section{Methods}

This cross-sectional study included 65 adult patients with confirmed SARS-CoV-2 infection admitted to the Critical Care Unit at the National Institute of Cardiology Ignacio Chavez from April 1,2020 , to June 20,2020. Informed consent was obtained from all individual participants included in the study. The study complied with the Declaration of Helsinki.

AKI was defined according to the KDIGO classification [11]. Clinical and laboratory data were obtained from the patient electronic medical records. The serum creatinine and urine output from ICU admission were registered and compared to meeting AKI criteria. The included patients were followed up until meeting AKI criteria, the start of RRT, or death. Mortality was defined as death of any cause during the follow-up (80 days).

\section{Image Acquisition}

\section{Image Acquisition and Quantification}

The protocol was designed to enable rapid image acquisition at the patient's bedside, and the image analysis was done outside the patient's room. The study was performed by an expert in critical care ultrasonography. The following measures were applied to minimize the exposure of the staff to the infection:

1. All studies were performed during regular/routine patient rounds.

2. The machine and sector probe were sanitized adequately between each evaluation.

3. All providers had adequate personal protective equipment.

4. Images were recorded in approximately $10 \mathrm{~min}$.

5. Measurements were performed outside the patient's room.

6. Analysis and registration of the measurements were performed by other physicians.

\section{Ultrasound Technique}

\section{Ultrasound Equipment}

The operator obtained the images using a phased array sector probe at $2-3 \mathrm{mHz}$, from the patient's right or left side, on any platform with the following modes: M-mode, 2D-mode, color, pulsed, continuous, and tissue Doppler. Sonographic equipment with advanced software technology was not an absolute requirement.

Comprehensive transthoracic echocardiography was performed according to guidelines $[12,13]$. The following echocardiographic views were obtained: parasternal long-axis view, parasternal short-axis view, apical 4-chamber view, apical 5-chamber view, and subcostal 4 -chamber view. The measured parameters were left ventricular (LV) diastolic diameter, LV outflow tract velocity-time integral, stroke volume, cardiac output, regional wall motion, mitral annular plane systolic excursion, LV ejection fraction, fractional shortening, tricuspid annular plane systolic excursion, tricuspid peak systolic S-wave tissue Doppler velocity, right ventricle (RV) basal diameter, and inferior vena cava (IVC) distensibility index (IVC diameter max - IVC diameter min/IVC diameter) $[14,15]$. Regarding lung ultrasound, the 12 points were evaluated according to the Oracle protocol [10].

Doppler ultrasound assesses arterial and venous blood flow velocity within the interlobar vessels of the kidney. From the arterial Doppler tracing, the RRI can be mea- 

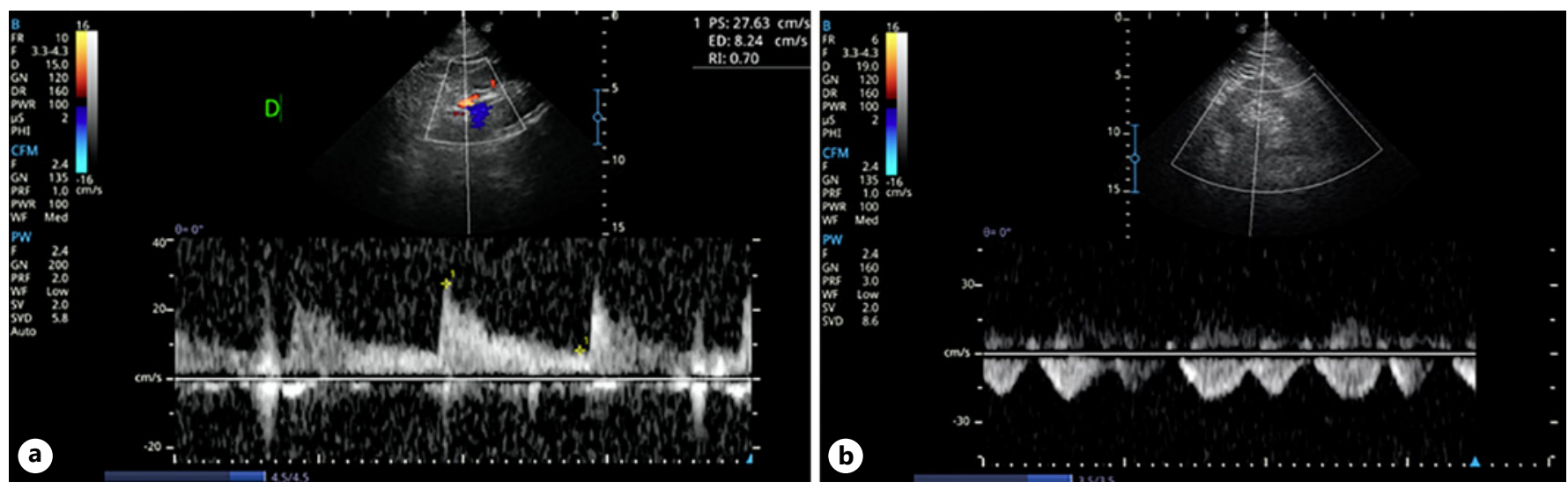

Fig. 1. a Doppler sample volume position in the interlobar vessels, intrarenal artery flow. b Pulsatile venous pattern.

sured from the maximal velocity $\left(V_{\max }\right)$ and the minimal velocity $\left(V_{\min }\right)$ during the cardiac cycle: $V_{\max }-V_{\min } / V_{\max }$. In addition, Doppler waveforms of intrarenal venous flow were divided into continuous, pulsatile, biphasic, and monophasic [16], as shown in Figure 1.

\section{Statistical Analysis}

Continuous variables were reported as mean and standard deviation, for normally distributed data, or median and interquartile range for those nonnormally distributed. Continuous variables were compared using the Kruskal-Wallis test or Student's $t$ test. Categorical variables were presented as frequencies and percentages, and we used the $\chi^{2}$ test and Fisher's exact test for comparisons, as appropriate according to the expected values. For the survival analysis, we used life tables and Kaplan-Meier survivor curves. We created a Cox proportional hazards model, adjusted for age and sex, to assess the variables that predicted mortality. All $p$ values $<0.05$ were considered statistically significant. All statistical analyses were performed using STATA V. 14.

\section{Results}

\section{Baseline Characteristics of the Population}

A cohort of 65 patients critically ill in the intensive care unit was included during the study period, $78.5 \%$ were males, and mean age was $53.4 \pm 12.5$. Diabetes and hypertension were present in $35.4 \%$ while we observed obesity and overweight in $63 \%$ of patients. The baseline characteristics are presented in Table 1 according to the RRI cutoff. Hypertension was the most prevalent in patients with $R R I \geq 0.7$. Serum creatinine and blood urea nitrogen were higher in patients with RRI $\geq 0.7$. Differences in Creactive protein, lactate, troponin, and potassium were observed between groups. No difference in ferritin, $\mathrm{pO}_{2}$, $\mathrm{HCO}_{3}$, fluid balance, urinary output, and days of hospital stay between groups was observed. D-dimer, $\mathrm{pCO}_{2}$, and NT-pro BNP showed a tendency to be higher in patients with $\mathrm{RRI} \geq 0.7$.

\section{Ultrasonographic Findings}

We found that the LV ejection fraction was higher in the group with RRI $<0.7(60 \%$, IQR 54-60) compared with the group with RRI $\geq 0.7$ (55\%, IQR 50-56) ( $p=$ $0.00)$. The LV outflow tract velocity-time integral variability was present in 31 patients $(47.7 \%)$, most of whom were in the RRI $<0.7$ group $(p=0.00)$. Only 10 patients (15.4\%) had RV dysfunction without differences between groups, and the IVC diameter was $18.6 \pm 3.3 \mathrm{~mm}$ in all patients, without differences between groups. Suprahepatic reverse flow was found in 9 patients (13.9\%), 2 in the $\mathrm{RRI}<0.7$ group and 7 in the RRI $\geq 0.7$ group $(p=0.02)$. We did not find a significant difference in the lung ultrasound score between groups. The pulsatile, biphasic, and monophasic venous patterns were significantly more frequent in patients with RRI $\geq 0.7$. The ultrasonographic findings are presented in Table 2.

\section{RRI and Outcomes}

In our cohort, 41 patients (63\%) were dependent of mechanical ventilation, 22 (54\%) developed AKI, 8 (12.3\%) required RRT, and $23(35.4 \%)$ died. We analyzed the ability of RRI to predict the outcomes. After analyzing RRI and outcomes, we found that most of the patients 
Table 1. Baseline clinical and laboratory characteristics according to RRI

\begin{tabular}{|c|c|c|c|c|}
\hline Females, $n(\%)$ & $14(21.5)$ & $10(25)$ & $4(16)$ & 0.390 \\
\hline Males, $n(\%)$ & $51(78.5)$ & $30(75)$ & $21(84)$ & \\
\hline Age, mean $\pm S D$, years & $53.4 \pm 12.5$ & $49.4 \pm 10.9$ & $59.8 \pm 12.4$ & 0.000 \\
\hline Diabetes, $n(\%)$ & $23(35.4)$ & $14(35)$ & $9(36)$ & 0.930 \\
\hline Hypertension, $n(\%)$ & $23(35.4)$ & $9(22.5)$ & $14(56)$ & 0.000 \\
\hline Smoking, $n(\%)$ & $11(16.9)$ & $5(12.5)$ & $6(24)$ & 0.310 \\
\hline Weight, median (IQR), kg & $78(70-85)$ & $80(68-87.5)$ & $75(70-83)$ & 0.720 \\
\hline Height, mean $\pm \mathrm{SD}, \mathrm{m}$ & $1.66 \pm 0.07$ & $1.67 \pm 0.07$ & $1.65 \pm 0.07$ & 0.440 \\
\hline Serum creatinine, median (IQR), mg/dL & $1.08(0.78-1.5)$ & $0.98(0.6-1.2)$ & $1.42(0.93-2.7)$ & 0.000 \\
\hline Blood urea nitrogen, median (IQR), mg/dL & $24.2(15-44)$ & $18.4(13.6-29.4)$ & $44.9(25-68)$ & 0.000 \\
\hline $\mathrm{K}$, median (IQR), mEq/L & $4.1(3.7-4.5)$ & $3.9(3.6-4.4)$ & $4.3(3.9-4.8)$ & 0.030 \\
\hline $\mathrm{Na}$, mean $\pm \mathrm{SD}, \mathrm{mEq} / \mathrm{L}$ & $135.9 \pm 5.7$ & $135.9 \pm 5.8$ & $135.9 \pm 5.7$ & 1 \\
\hline D-dimer, median (IQR), ng/mL & $0.54(0.3-1.07)$ & $0.41(0.25-0.96)$ & $0.61(0.43-1.5)$ & 0.080 \\
\hline C-reactive protein, median (IQR), mg/L & $177(87-252)$ & $147(71-197)$ & $222(158-352)$ & 0.000 \\
\hline $\mathrm{pH}$, median (IQR) & $7.42(7.35-7.46)$ & $7.42(7.40-7.47)$ & $7.37(7.28-7.42)$ & 0.000 \\
\hline $\mathrm{pO}_{2}$, mean $\pm \mathrm{SD}$ & $65(49-78)$ & $65(51-79)$ & $64(43-76)$ & 0.360 \\
\hline $\mathrm{pCO}_{2}$, median (IQR) & $35(30-40)$ & $34(29-37)$ & $37(33-47)$ & 0.070 \\
\hline $\mathrm{HCO}_{3}$, median (IQR) & $22(19-25)$ & $22(19-25)$ & $22(18-24)$ & 0.940 \\
\hline Lactate, median (IQR) & $1.5(1.2-2.4)$ & $1.4(1.1-2)$ & $2.2(1.3-3.3)$ & 0.010 \\
\hline Net fluid balance, median (IQR), mL & $-77(-741$ to 380$)$ & $-80(-610$ to 356$)$ & $-77(-844$ to 404$)$ & 0.860 \\
\hline Urinary output, median (IQR), mL & $0.74(0.5-0.9)$ & $0.8(0.5-0.9)$ & $0.6(0.2-0.9)$ & 0.160 \\
\hline In-hospital stay, median (IQR), days & $15(10-26)$ & $14(10-26)$ & $15(11-24)$ & 0.890 \\
\hline
\end{tabular}

RRI, renal resistive index; SD, standard deviation; IQR, interquartile range.

Table 2. Ultrasonographic findings

\begin{tabular}{|c|c|c|c|c|}
\hline Variable & Total & $\mathrm{RRI}<0.7$ & $\mathrm{RRI} \geq 0.7$ & $p$ value \\
\hline \multicolumn{5}{|l|}{ Right venous pattern, $n(\%)$} \\
\hline Continuous & $34(52.3)$ & $33(82.5)$ & $1(4)$ & 0.000 \\
\hline Pulsatile & $12(18.5)$ & $3(7.5)$ & $9(36)$ & \\
\hline Biphasic & $14(21.5)$ & $4(10)$ & $10(40)$ & \\
\hline Monophasic & $5(7.7)$ & 0 & $5(20)$ & \\
\hline \multicolumn{5}{|l|}{ Left venous pattern, $n(\%)$} \\
\hline Pulsatile & $11(16.9)$ & $3(7.5)$ & $8(32)$ & \\
\hline Biphasic & $15(23.1)$ & $3(7.5)$ & $12(48)$ & \\
\hline Monophasic & $4(6.2)$ & 0 & $4(16)$ & \\
\hline Suprahepatic reverse flow & $9(13.9)$ & $2(5)$ & $7(28)$ & 0.020 \\
\hline IVC diameter (min), mean $\pm \mathrm{SD}, \mathrm{mm}$ & $13.6 \pm 4.3$ & $13.2 \pm 4$ & $14.2 \pm 4.7$ & 0.350 \\
\hline IVC diameter $(\max )$, mean $\pm \mathrm{SD}, \mathrm{mm}$ & $18.6 \pm 3.3$ & $18.4 \pm 3$ & $18.8 \pm 3.7$ & 0.580 \\
\hline
\end{tabular}

RRI, renal resistive index; IVC, inferior vena cava; SD, standard deviation; IQR, interquartile range; LVEF, left ventricular ejection fraction; LUS, lung ultrasound; LVOT VTI, left ventricular outflow tract velocity-time integral; RV, right ventricle. 
Fig. 2. Kaplan-Meier survival estimates according to RRI. RRI, renal resistive index.

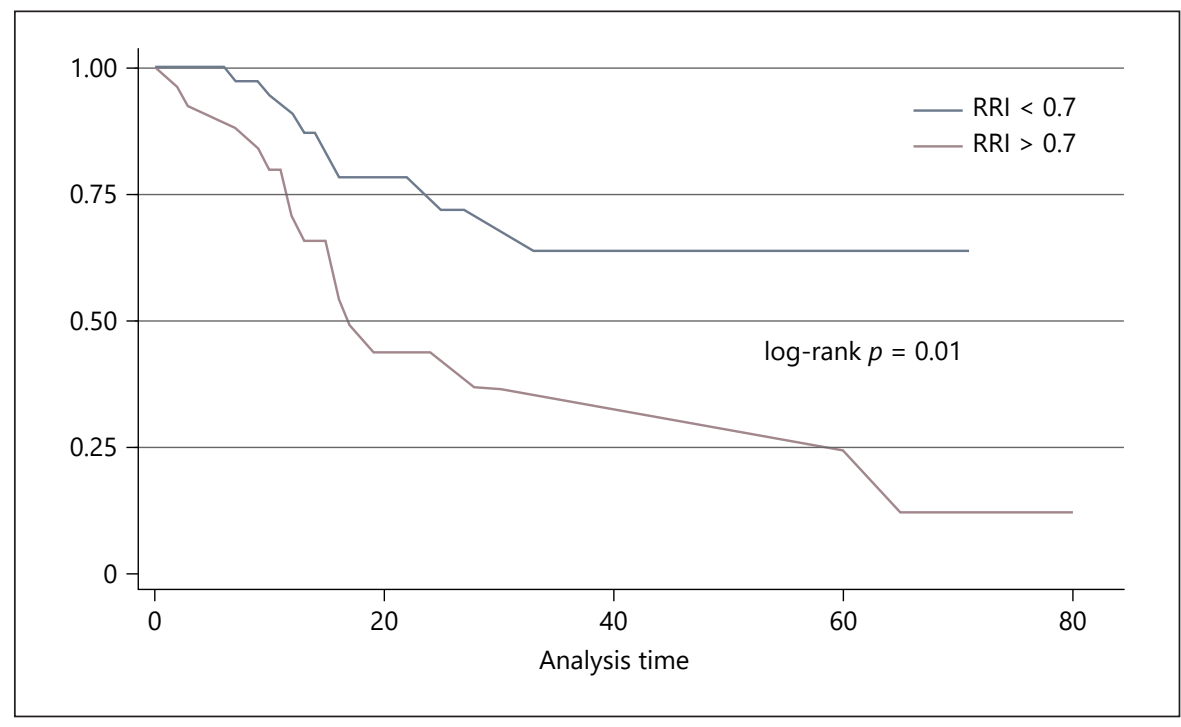

Table 3. RRI and outcomes

\begin{tabular}{|c|c|c|c|c|}
\hline Outcome & Total & $\mathrm{RRI}<0.7$ & $\mathrm{RRI} \geq 0.7$ & $p$ value \\
\hline Acute kidney injury, $n(\%)$ & $22(33.9)$ & $7(32)$ & $15(68)$ & 0.000 \\
\hline Renal replacement therapy, $n(\%)$ & $8(12.3)$ & $2(25)$ & $6(75)$ & 0.030 \\
\hline Mechanical ventilation, $n(\%)$ & $41(63.1)$ & $22(54)$ & $19(46)$ & 0.080 \\
\hline Mortality, $n(\%)$ & $23(35.4)$ & $8(35)$ & $15(65)$ & 0.000 \\
\hline
\end{tabular}

RRI, renal resistive index.

Table 4. Cox regression model adjusted by age and sex for mortality

\begin{tabular}{lcccc}
\hline Variable & HR & SE & $p$ value & $95 \%$ CI \\
\hline Age & 1.03 & 0.02 & 0.070 & $0.99-1.08$ \\
Sex & 1.22 & 0.68 & 0.720 & $0.40-3.64$ \\
RRI $\geq 0.7$ & 2.86 & 1.26 & 0.010 & $1.19-6.82$ \\
Diabetes & 0.82 & 0.36 & 0.660 & $0.34-1.96$ \\
Hypertension & 1.29 & 0.58 & 0.560 & $0.53-3.13$ \\
Overweight/obesity & 0.63 & 0.26 & 0.270 & $0.27-1.44$ \\
\hline
\end{tabular}

RRI, renal resistive index.

who developed AKI and required RRT had RRI $\geq 0.7$. Also, $65 \%$ of patients who died had RRT $\geq 0.7$. RRI $\geq 0.7$ showed a trend to have a higher risk for mechanical ventilation. These outcomes are shown in Table 3.

\section{RRI and Survival}

Analysis using Kaplan-Meier curves of the 2 different groups was divided according to RRI. Having RRI $\geq 0.7$ was associated with lower survival at follow-up as shown in Figure 2. In the Cox model adjusted for the potential confounding variables listed above, the RRI $\geq 0.7$ was associated with higher mortality (HR 2.86, 95\% CI: 1.19 $6.82, p=0.01)$ as presented in Table 4 .

\section{Discussion}

Point-of-care ultrasound allowed a bedside evaluation with real-time evaluation [11]. RRI has become part of the organ function assessment using Doppler ultrasound [12]. The RRI is derived from intrarenal Doppler waveforms as ([peak systolic velocity - end-diastolic velocity]/ peak systolic velocity) at the renal segmental artery [13]. Previously, RRI was considered to reflect only intrarenal vascular pathological processes; however, currently, RRI is a tool for assessing hemodynamics and changes in kidney perfusion in critically ill patients $[17,18]$.

Some of the proposed uses of RRI in AKI have been to assess kidney perfusion, predict kidney response to 
vasoactive medications, and predict the onset and recovery of AKI [19]. RRI may be secondary to a decrease in mixed venous oxygen saturation, reflecting an early vascular response to tissue hypoxia [20]. Also, the ability of RRI to discriminate a prerenal cause of acute tubular necrosis in AKI stage 3 had previously been described [21]. However, AKI is a continuum where the etiological mechanism, severity, and duration of the injury determine the histological and clinical consequences [22]. Renal and extrarenal factors can influence the RRI value [23].

Since the Lerolle et al. [24] study, the RRI was described as a predictor for developing AKI in critically ill patients. Haitsma-Mulier et al. [25] prospectively studied 99 critically ill patients and reported that at admission, RRI in patients developing AKI, including severe AKI, was higher compared with patients not developing AKI (0.71 vs. 0.65$)$. Schnell et al. [26] evaluated 58 patients with severe sepsis or polytrauma and found that RRI was the only parameter predictive of severe AKI compared with serum and urine cystatin C. In the meta-analysis of 9 studies by Ninet et al. [27], evaluating the performance of RRI in AKI reversibility, they found a strong association between higher RRI and persistent AKI (OR, 29.85, 95\% CI: 8.73-102.16; $p<0.00001$ ). Darmon et al. [28] reported that higher RRI has predictive value for mechanical ventilation. However, Wiersema et al. [29] reported a limited diagnostic accuracy of renal perfusion by renal ultrasound for AKI.

Renberg et al. [30] reported for the first time the usefulness of measuring RRI in patients with COVID-19. They found that RRI was higher in patients with AKI compared to patients without AKI. Furthermore, RRI was higher in oliguric patients than in nonoliguric patients.

Our study is the first to investigate the predictive power of RRI in the incidence of AKI, mechanical ventilation dependence, renal replacement therapy initiation, and mortality. Interestingly, LV dysfunction was significantly more frequent in patients with $R R I \geq 0.7$, which contrasts with previously reported [31]. Although RV dysfunction and IVC diameter were not different between groups, suprahepatic reverse flow was more frequent significantly in RRI $\geq 0.7$, which supports the venous congestion paradigm. Venous congestion can produce elevated right-side pressures that are transmitted to the hepatic and renal venous flow that increases the pulsatility of the intrarenal venous flow. This pulsatility could show peaks during each cardiac cycle because of biphasic velocity (biphasic pattern) or a diastolic only flow (mono- phasic pattern) secondary to disminution of systolic velocity [32]. Lida et al. [33] showed that monophasic venous flow was associated with RRT and corroborated a significant increase in renal congestion. Likewise, Maeder et al. [34] mentioned that the increase in central venous pressure and renal venous pressure could probably be the cause of these findings. In this sense, we found that the monophasic venous pattern was associated with mortality.

Direct association between RRI and central or peripheral pulse pressure has been shown because increased pulsatile stress causes endothelial damage in small arteries [35]. It has been suggested that increased RRI is a marker of subclinical target organ damage [36].

We found that patients with RRI $\geq 0.7$ had more incidence of AKI, renal replacement therapy requirement, and mortality and higher trend to mechanical ventilation requirement. Also, RRI $\geq 0.7$ was predictive for higher mortality and less survival.

Deleterious interactions between kidney and lung dysfunctions could have many mechanisms: positive-pressure ventilation, hypoxemia, and systemic inflammation. Positive-pressure ventilation decreases GFR, renal blood flow, and free water clearance. Hypoxemia and hypercapnia modify renal vascular resistances and increase diuresis and lead to kidney injury. Also, COVID-19 patients have elevated level of inflammatory cytokines that interact with kidney resident cells and induce endothelial and tubular dysfunction $[37,38]$.

Our study has a few limitations. First, the number of patients is small due to the technical difficulties of treating these patients. Second, AKI diagnosis was made according to the KDIGO guidelines, with its known drawbacks. Third, RRI is affected by several factors other than the kidney and systemic hemodynamics. Finally, because a single RRI measurement was performed, the association with recovery of renal function was not studied.

Despite these limitations, our study has a number of strengths. First, RRI measurements were performed by an expert in critical care ultrasonography. Second, although Renberg et al. [30] investigated the association between RRI and AKI diagnosis, AKI severity, and urinary output, this study is the first to evaluate RRI as a predictor tool of outcomes including mechanical ventilation, RRT initiation, and mortality in patients with COVID-19. We considered that our findings have implications for the application of early interventions. However, our results need to be further validated in cohorts. 


\section{Conclusion}

The RRI measured by ultrasound is a noninvasive, reproducible, and accurate bedside method. This study showed that an RRI $\geq 0.7$ predicts a higher risk of AKI, need for RRT, and mortality in patients with severe SARSCoV-2 pneumonia. From this study, it could be evaluated in controlled studies if the RRI value is associated with kidney damage due to COVID-19 or is due to other causes such as heart failure and sepsis.

\section{Statement of Ethics}

Written informed consent was obtained at admission from the patients or relatives. The need for approval by the institutional ethics committee was not required due to the retrospective nature of the study. However, the study complied with the Declaration of Helsinki.

\section{Conflict of Interest Statement}

In the last 3 years, Claudio Ronco has been consulting or is a part of the advisory boards for ASAHI, Astute, Baxter, Biomerieux, B. Braun, Cytosorbents, ESTOR, FMC, GE, Jafron, Medtronic, and Toray. The other authors have no conflicts of interest to declare.

\section{Funding Sources}

This research received no specific grant from any funding agency from any sector.

\section{Author Contributions}

Edgar Garcia Cruz: conceptualization and writing - original draft; Blanca Estela Broca Garcia: investigation and writing original draft - review and editing; Enzo Vasquez Jimenez: investigation and writing original draft - review and editing; Daniel Manzur Sandoval: investigation and writing - review and editing; Rodrigo Gopar Nieto: methodology, review and editing; Francisco Javier Gonzalez Ruiz: investigation and review and editing; Magdalena Madero: methodology, writing - review and editing; Linda Diaz Gallardo: investigation and writing - review and editing; Claudio Ronco: review and editing.

\section{References}

1 Guan WJ, Ni ZY, Hu Y, Liang WH, Ou CQ, $\mathrm{He} \mathrm{XX}$, et al. Clinical characteristics of coronavirus disease 2019 in China. N Engl J Med. 2020 Apr 30;382(18):1708-20.

2 Cheng Y, Luo R, Wang K, Zhang M, Wang Z, Dong $\mathrm{L}$, et al. Kidney disease is associated with in-hospital death of patients with COVID-19. Kidney Int. 2020;97(5):829-38.

3 Ronco C, Reis T, Husain-Syed F. Management of acute kidney injury in patients with COVID-19. Lancet Respir Med. 2020;8(7): $738-42$.

4 Diao B, Wang C, Wang R, Feng Z, Tan Y, Wang $\mathrm{H}$, et al. Human kidney is a target for novel severe acute respiratory syndrome coronavirus 2 (SARS-CoV-2) infection. medRxiv. 2020.

5 Ronco C, Reis T. Kidney involvement in COVID-19 and rationale for extracorporeal therapies. Nat Rev Nephrol. 2020;16(6):308-10.

6 Gabarre P, Dumas G, Dupont T, Darmon M, Azoulay E, Zafrani L. Acute kidney injury in critically ill patients with COVID-19. Intensive Care Med. 2020;46(7):1339-48.

7 Li Z, Wu M, Yao J, Guo J, Liao X, Song S, et al. Caution on kidney dysfunctions of COVID-2019 patients. medRxiv. 2020.

8 Joannidis M, Forni LG, Klein SJ, Honore PM, Kashani K, Ostermann M, et al. Lung-kidney interactions in critically ill patients: consensus report of the acute disease quality initiative (ADQI) 21 workgroup. Intensive Care Med. 2020;46(4):654-72.
9 Cauwenberghs N, Kuznetsova T. Determinants and prognostic significance of the renal resistive index. Pulse. 2016;3(3-4):172-8.

10 García-Cruz E, Manzur-Sandoval D, RascónSabido R, Gopar-Nieto R, Barajas-Campos RL, Jordán Ríos A, et al. Critical care ultrasonography during COVID-19 pandemic: the ORACLE protocol. Echocardiography. 2020; 37(9):1353-61.

11 Kidney Disease: Improving Global Outcomes (KDIGO) Acute Kidney Injury Work Group. KDIGO clinical practice guideline for acute kidney injury. Kidney inter Suppl. 2012;2:1138.

12 Moore CL, Copel JA. Point-of-care ultrasonography. N Engl J Med. 2011;364(8):749-57.

13 Ponte B, Pruijm M, Ackermann D, Vuistiner $P$, Eisenberger U, Guessous I, et al. Reference values and factors associated with renal resistive index in a family-based population study. Hypertension. 2014;63(1):136-42.

14 Lang RM, Badano LP, Mor-Avi V, Afilalo J, Armstrong A, Ernande L, et al. Recommendations for cardiac chamber quantification by echocardiography in adults: an update from the American society of echocardiography and the European association of cardiovascular imaging. Eur Heart J Cardiovasc Imaging. 2015;16(1):233-70

15 Mitchell C, Rahko PS, Blauwet LA, Canaday B, Finstuen JA, Foster MC, et al. Guidelines for performing a comprehensive transthoracic echocardiographic examination in adults: recommendations from the American society of echocardiography. J Am Soc Echocardiogr. 2019;32(1):1-64.

16 Platt JF, Ellis JH, Rubin JM, DiPietro MA, Sedman AB. Intrarenal arterial doppler sonography in patients with nonobstructive renal disease: correlation of resistive index with biopsy findings. AJR Am J Roentgenol. 1990; 154(6):1223-7.

17 Tublin ME, Bude RO, Platt JF. Review. The resistive index in renal doppler sonography: where do we stand? AJR Am J Roentgenol, 2003;180(4):885-92.

18 Le Dorze M, Bouglé A, Deruddre S, Duranteau J. Renal doppler ultrasound: a new tool to assess renal perfusion in critical illness. Shock. 2012;37(4):360-5.

19 Spatola L, Andrulli S. Doppler ultrasound in kidney diseases: a key parameter in clinical long-term follow-up. J Ultrasound. 2016; 19(4):243-50.

20 Corradi F, Brusasco C, Paparo F, Manca T, Santori G, Benassi F, et al. Renal doppler resistive index as a marker of oxygen supply and demand mismatch in postoperative cardiac surgery patients. Biomed Res Int. 2015;2015: 763940.

21 Pozzi Mucelli R, Bertolotto M, Quaia E. Imaging techniques in acute renal failure. Contrib Nephrol. 2001(132):76-91.

22 Schneider AG, Bellomo R. Urinalysis and prerenal acute kidney injury: time to move on Crit Care. 2013;17(3):141. 
23 Di Nicolo P, Granata A. Renal intraparenchymal resistive index: the ultrasonographic answer to many clinical questions. J Nephrol. 2019;32(4):527-38.

24 Lerolle N, Guérot E, Faisy C, Bornstain C, Diehl JL, Fagon JY. Renal failure in septic shock: predictive value of doppler-based renal arterial resistive index. Intensive Care Med. 2006;32(10):1553-9.

25 Haitsma Mulier JLG, Rozemeijer S, Röttgering JG, Spoelstra-de Man AME, Elbers PWG, Tuinman PR, et al. Renal resistive index as an early predictor and discriminator of acute kidney injury in critically ill patients; A prospective observational cohort study. PLoS One. 2018;13(6): $\mathrm{e} 0197967$.

26 Schnell D, Darmon M. Bedside doppler ultrasound for the assessment of renal perfusion in the ICU: advantages and limitations of the available techniques. Crit Ultrasound J. 2015; $7(1): 24$.

27 Ninet S, Schnell D, Dewitte A, Zeni F, Meziani F, Darmon M. Doppler-based renal resistive index for prediction of renal dysfunction reversibility: a systematic review and metaanalysis. J Crit Care. 2015;30(3):629-35.
28 Darmon M, Schortgen F, Vargas F, Liazydi A, Schlemmer B, Brun-Buisson C, et al. Diagnostic accuracy of doppler renal resistive index for reversibility of acute kidney injury in critically ill patients. Intensive Care Med. 2011;37(1):68-76.

29 Wiersema R, Kaufmann T, van der Veen HN, de Haas RJ, Franssen CFM, Koeze J, et al. Diagnostic accuracy of arterial and venous renal doppler assessment for acute kidney injury in critically ill patients: a prospective study. J Crit Care. 2020;59:57-62.

30 Renberg M, Jonmarker O, Kilhamn N, RimesStigare C, Bell M, Hertzberg D. Renal resistive index is associated with acute kidney injury in COVID-19 patients treated in the ICU. 2020 Sep 15, PREPRINT (Version 1) available at Research Square.

31 Kuznetsova T, Cauwenberghs N, Knez J, Thijs L, Liu YP, Gu YM, et al. Doppler indexes of left ventricular systolic and diastolic flow and central pulse pressure in relation to renal resistive index. Am J Hypertens. 2015;28(4): $535-45$.

32 Tang WH, Kitai T. Intrarenal venous flow: window into the congestive kidney failure phenotype of heart failure? JACC Heart Fail. 2016;4(8):683-6.
33 Lida N, Seo Y, Sai S, Machino-Ohtsuka T, Yamamoto M, Ishizu T, et al. Clinical implications of intrarenal hemodynamic evaluation by doppler ultrasonography in heart failure. JACC Heart Fail. 2016;4(8):674-82.

34 Maeder MT, Holst DP, Kaye DM. Tricuspid regurgitation contributes to renal dysfunction in patients with heart failure. J Card Fail. 2008;14(10):824-30.

35 O'Rourke MF, Safar ME. Relationship between aortic stiffening and microvascular disease in brain and kidney: cause and logic of therapy. Hypertension. 2005;46(1):200-4.

36 Doi Y, Iwashima Y, Yoshihara F, Kamide K, Takata H, Fujii T, et al. Association of renal resistive index with target organ damage in essential hypertension. Am J Hypertens. 2012;25(12):1292-8.

37 Hildebrandt W, Ottenbacher A, Schuster M, Swenson ER, Bärtsch P. Diuretic effect of hypoxia, hypocapnia, and hyperpnea in humans: relation to hormones and $\mathrm{O}(2)$ chemosensitivity. J Appl Physiol. 2000;88(2):599-610.

38 Darmon M, Clec'h C, Adrie C, Argaud L, Allaouchiche B, Azoulay E, et al. Acute respiratory distress syndrome and risk of AKI among critically ill patients. Clin J Am Soc Nephrol. 2014;9(8):1347-53. 